\title{
Molecular dynamics simulations exploring the interaction between DNA and metalated bleomycin
}

\author{
Viraja R. Palwai ${ }^{1}$, Leif A. Eriksson ${ }^{2^{*}}$ \\ ${ }^{1}$ Örebro Life Science Center, School of Science and Technology, Örebro University, Örebro, Sweden; \\ ${ }^{2}$ School of Chemistry, National University of Ireland, Galway, Ireland; *Corresponding Author: leif.eriksson@nuigalway.ie
}

Received 25 February 2011; revised 27 March 2011; accepted 4 April 2011.

\begin{abstract}
Bleomycin (BIm) is a natural antibiotic with antitumour activity, used as a combination drug in treatment of various types of cancers. BIm intercalates with DNA and will in the presence of a redox metal ion and molecular oxygen form an activated bleomycin complex capable of releasing free radicals and subsequently leading to DNA cleavage. The present theoretical work was carried out to better understand the interaction between DNA and BIm using different metal co-factors ( $\mathrm{Co}$ and $\mathrm{Fe}$ ). Binding energies and structural properties were analysed for both the complexes. The results show that BIm binds stronger to DNA when complexed with Fe, and provides a better structural orientation compared to the CoBIm complex in order to abstract the H4' hydrogen of deoxyribose that initiates the DNA strand cleavage process. The short distance between the iron-bound peroxide and the deoxyribose $\mathrm{H}^{4}$ ' furthermore supports the previously proposed direct abstraction mechanism.
\end{abstract}

Keywords: DNA; Bleomycin; Molecular dynamics; Binding energy; Strand break

\section{INTRODUCTION}

Drugs interacting with DNA has been a subject of interest for many years due to their therapeutic value as anti-cancer agents [1]. Drugs targeting DNA are largely classified into two types: minor groove binding and intercalating, respectively. Minor groove binders, for example netropsin, do not generate any large structural deformations of DNA [2], whereas, intercalators show their therapeutic activity by lengthening and unwinding of DNA, such as for example daunomycin [3,4]. Bleomycin (Blm, Figure 1) both intercalates and binds to the minor groove [3], and thus displays a combination of the above features.

Blm is a natural product that belongs to the family of glycopeptide antibiotics obtained from Streptomyces verticillus [5]. It is used in combination with other chemotherapeutic drugs to treat various types of cancers such as Hodgkin's lymphoma and cancers of skin, head and neck [6,7]. The structure of Blm mainly consists of four domains (Figure 1): the metal binding domain resides in the minor groove and is responsible for oxygen activation and subsequent hydrogen abstraction from DNA. The bithiazole tail shows partial intercalation, and the C-terminal sulphonium subunit interacts with the major groove. Both these subunits contribute to DNA binding [8]. The linker region plays an important role in DNA cleavage [9], whereas the carbohydrate domain sits above the metal binding domain in the minor groove [8]. Most of the natural Blm's like phleomycin, pleomycin, Blm $A_{2}$ and Blm $B_{2}$ differ mainly in the bithiazole tail moiety [10].

The cytotoxicity of Blm is related to its ability to cleave single and double stranded DNA, the latter being difficult for the cell to repair [11]. In vivo, Blm in the presence of its co-factors Fe and oxygen forms an activated bleomycin complex [12]. The activated bleomycin in turn abstracts a hydrogen atom from a backbone deoxyribose sugar of DNA, and thereby generating a sugar centered radical. This radical reacts further by cleaving the DNA strand or releasing free nucleobases [13-15]. In particular pyrimidine nucleotides in the 5'GC and 5'GT sequences are preferred sites of attack [11].

Several structures of metalated Blm's bound to DNA are available with Co (III) or Ga (III) or as complexing metal [16-18]. To our knowledge no NMR structures are available of Blm in complex with iron, due to the paramagnetic nature of the ferrous ion Fe (III) present in the activated Blm in vivo, which makes it difficult to detect using NMR [19]. In addition, there are differences in reactivity between FeBlm and CoBlm that also contribute towards the difference in detectability. Studies have shown that Blm exerts greater affinity to chelate with $\mathrm{Fe}$ 


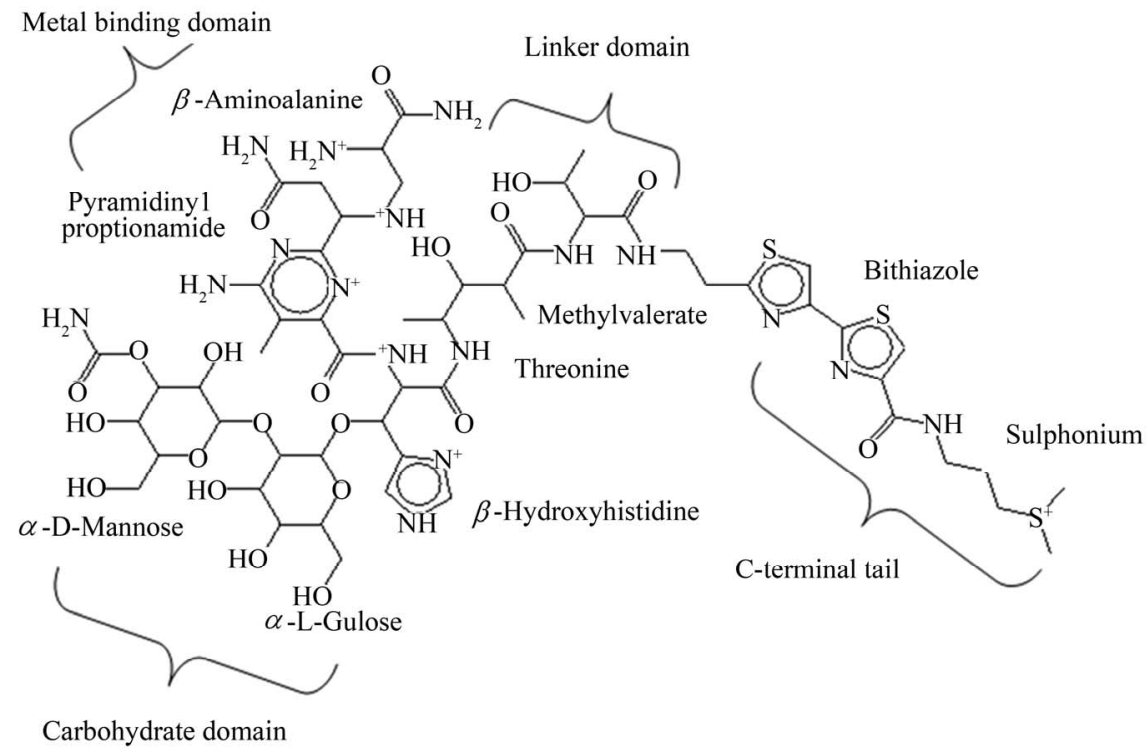

Figure 1. Structure of bleomycin $A_{2}$. Metal coordinating ligands are indicated with an asterisk $\left(^{*}\right)$.

than Co, and that FeBlm displays higher antitumor (DNA cleaving) activity compared to CoBlm [20,21]. Despite this, the activated $\mathrm{Co}(\mathrm{III}) \mathrm{Blm}-\mathrm{OOH}$ complex is often considered an analogous system to Fe(III)Blm$\mathrm{OOH}$, based on the structural correlation and similar binding modes [19].

Previous theoretical studies of Blm have been carried out using Density Functional Theory (DFT) to study possible reaction mechanisms of activated bleomycin (Blm-Fe(III)OOH). Three reaction pathways for activated bleomycin to cleave single and double stranded DNA has been proposed in absence of crystal/NMR structures; a) heterolytic cleavage in which Blm-Fe(III) $\mathrm{OOH}$ undergoes heterolytic cleavage of the $\mathrm{O}-\mathrm{OH}$ bond and leads to formation of $\mathrm{Blm}-\mathrm{Fe}(\mathrm{V})=\mathrm{O}$ and water; b) homolytic cleavage of $\mathrm{O}-\mathrm{OH}$ which leads to formation of $\mathrm{Blm}-\mathrm{Fe}(\mathrm{IV})=\mathrm{O}$ and an oxygen centered hydroxyl radical that in turn abstracts the $\mathrm{H} 4$ ' hydrogen; $\mathrm{c}$ ) direct hydrogen atom abstraction from the adjacent dexoyribose sugar leading to formation of $\mathrm{Blm}-\mathrm{Fe}(\mathrm{IV})=\mathrm{O}$, water and sugar centered radical [22]. According to the study carried out by Decker et al, the first two pathways are ruled out as they are endergonic and energetically unfavorable. The third pathway, i.e., direct hydrogen abstraction is shown to be exergonic and overall more favorable over the other two [22]. In another theoretical study the first pathway was shown to be more favorable [23]. The difference between the results in the two studies was attributed to different methodology employed. Further work has, however, provided support for the third, direct, pathway [24]. Theoretical studies have also been carried out to explore the nature of the sixth axial ligand of the metal binding domain [25].
The present study was carried out to explore the interaction of Blm with DNA when complexed with Co and $\mathrm{Fe}$, using molecular mechanics/molecular dynamics based methods. In particular, structural differences between the two activated complexes are studied, as well as properties such as binding energies and electrostatic surfaces, in order to provide insight on the drug-DNA interactions and to try to provide a rationale behind the higher reactivity of activated FeBlm and the potential reaction pathway.

\section{COMPUTATIONAL DETAILS}

All modeling and molecular dynamics (MD) simulations were performed using YASARA [26]. The structure of the dsDNA sequence d(GAGCTC) ${ }_{2}$ complexed with Co(III) Blm, PDB ID 1MTG [27], was retrieved from the Protein Data Bank. Another NMR structure with PDB ID 1MXK; CoBlm complexed with d(GGAAGCTTCC $)_{2}$, is also available. Based on initial benchmarking to explore structure and stability of the two metalated complexes, the smaller system was chosen for the full study in order to enable faster calculations. All simulations were carried out using the AMBER 03 force field [28]. Two complexes were prepared for MD simulations, CoBlm-DNA and FeBlm-DNA. For the second complex, Co was substituted by Fe using the same oxidation state (III) as in the Co containing crystal structure. Long-range interactions were treated with the Particle Mesh Ewald algorithm [29] with a cut off value of $7.86 \AA$. Periodic boundary conditions were defined using a water filled cell of dimensions $44 \times 44 \times 44 \AA$ for the FeBlm system and $43 \times 48 \times 41 \AA$ for the CoBlm 
system. Each DNA-Blm system was solvated with TIP3P [30] water molecules. Counter ions were randomly placed in the box to neutralize the DNA and the positively charged Blm. For the CoBlm-DNA system, twelve $\mathrm{Na}^{+}$, three $\mathrm{Cl}^{-}$and 2688 water molecules were added making it a 8640 atom system. The FeBlm system contained slightly more water molecules giving a total of 8893 atoms.

Each system was subjected to steepest descent minimization-this is hereafter referred to as the initial structure. After removing conformational stress through equilibration simulations (referred to as the equilibrated structure), production simulations were carried out with a time step of $0.5 \mathrm{fs}$ and with snapshots saved in the trajectory every 3 ps. All simulations were carried out at constant pressure and temperature (NPT) conditions at 298K, for a period of 25 ns. After the 25 ns MD simulations, the structures were minimized again, giving the final structures. Root mean square deviations (RMSD) were computed between the different systems and structures, as discussed in the following.

The labelling of DNA bases are displayed in Supplementary material, Figure S1; labels of atoms involved in hydrogen bonded interactions are shown in Figure S2; and atomic labelling of the nucleobases in Figure S3.

\section{RESULTS AND DISCUSSION}

MD simulations for 25 ns resulted in 8333 snapshots for each system. The stabilities of the trajectories were analysed by measuring the RMSD of the DNA backbone, cf. Figure 2. The sudden drift in the RMSD for CoBlm between 3 and 4 ns is due to motion of one of the terminal basepairs, which returned to the normal position after 1 ns and remained there until the end of the simulation. Both systems were considered equilibrated after $5 \mathrm{~ns}$.

\subsection{Structural Changes in BIm and DNA}

The RMSDs between the energy minimized initial and final structures of the Blm molecules, DNA, and the complete systems, are listed in Table 1. For both complexes, the distances between the metal and the metal binding ligands were measured; no differences were observed indicating that the metal binding as such was stable. In the two systems, the final structures after MD simulation display slightly different orientations of the terminal tail in that the sulphonium region points downwards in the CoBlm complex (Figure 3(a)) and upwards in the FeBlm complex (Figure 3(b)). The tails furthermore displace further into the major groove when compared to the initial positions of CoBlm obtained from NMR spectroscopy. The intercalated region, the carbohydrate domain and the linker region do, however, not show any large structural differences, although in CoBlm the carbohydrate domain moves back slightly towards the terminal base pairs in the final structure (Figure 3(a)). No major changes were observed in the other parts of CoBlm. The heavy atom RMSDs between the starting (NMR) structure and energy minimized initial structures were found to be only $0.5 \AA$ for both systems, and they also display very similar binding modes (not shown).

The equilibrated structures (after 5 ns MD) were also compared to the final structures (25 ns MD) to check for any structural changes that may have occurred during the production simulations. CoBlm displayed some variation in the orientation of the Blm molecule. The carbohydrate binding domain was initially positioned away from the terminal base pairs, whereas for the equilibrated structure this tilts back towards the terminal base pairs, although it does not show any direct interaction with DNA. This position is then retained throughout the simulation

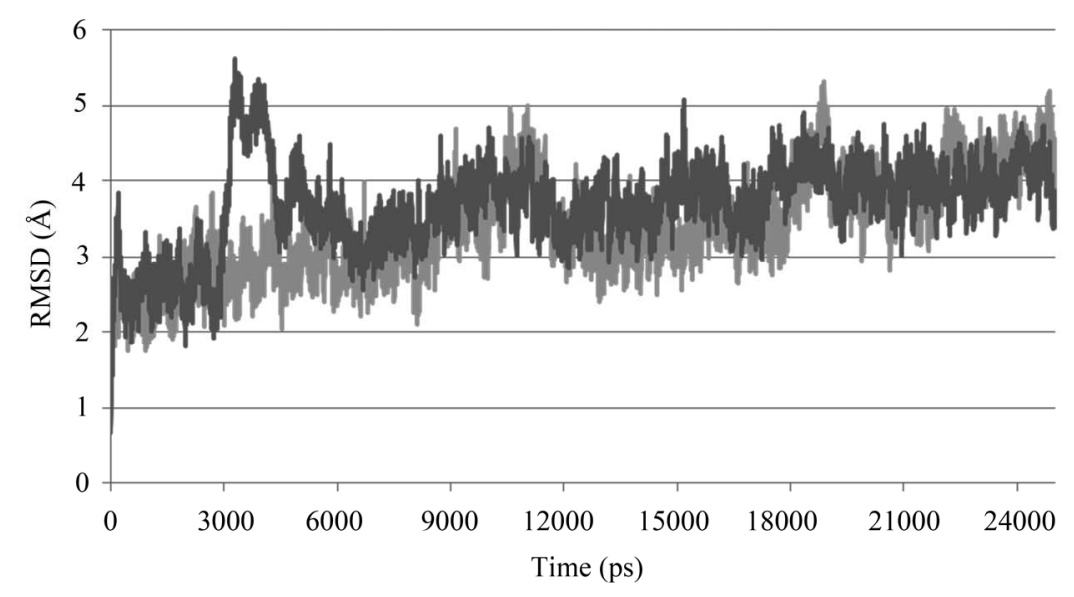

Figure 2. RMSD of DNA backbone versus time during the MD simulation. CoBlm in black and FeBlm in grey. 
Table 1. RMSD (heavy atoms) in $\AA$, between the energy minimised initial structures and final structures after $25 \mathrm{~ns}$ MD, for DNA, MeBlm and full system; ${ }^{a}$ and Blm Binding Energy in final system $(\mathrm{kcal} / \mathrm{mol})$.

\begin{tabular}{ccccc}
\hline Complex & RMSD $(\AA)$ & DNA & $\begin{array}{c}\text { MeBlm Full } \\
\text { System }\end{array}$ & $\begin{array}{c}\text { Binding Energy } \\
(\mathrm{kcal} / \mathrm{mol})\end{array}$ \\
\hline CoBlm & $2.8(2.4)$ & $2.4(2.1)$ & $2.9(2.3)$ & 405.37 \\
FeBlm & $3.6(3.0)$ & $2.0(1.9)$ & $3.3(2.7)$ & 720.55 \\
\hline
\end{tabular}

${ }^{\mathrm{a}}$ Values in parentheses: RMSD between energy minimized equilibrated and final structures.

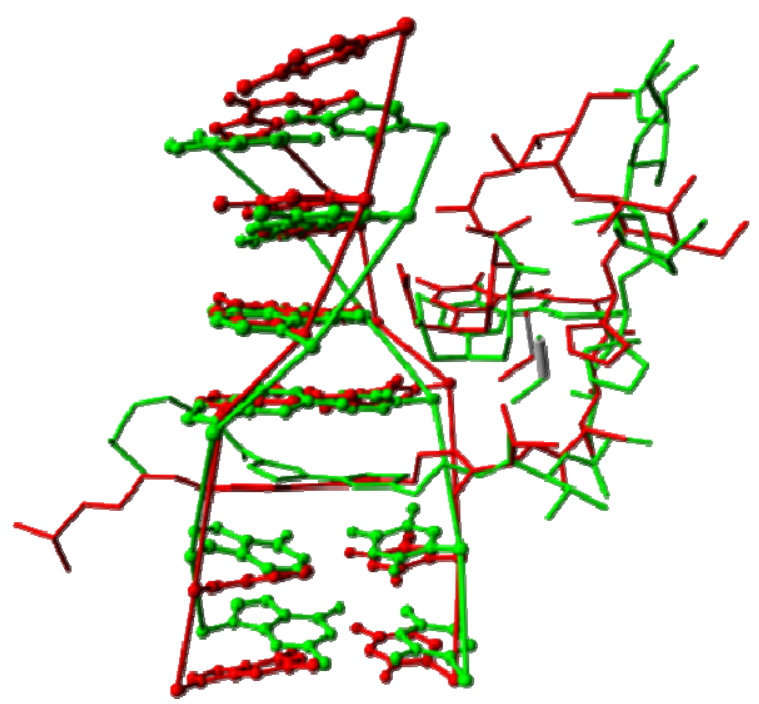

(a)

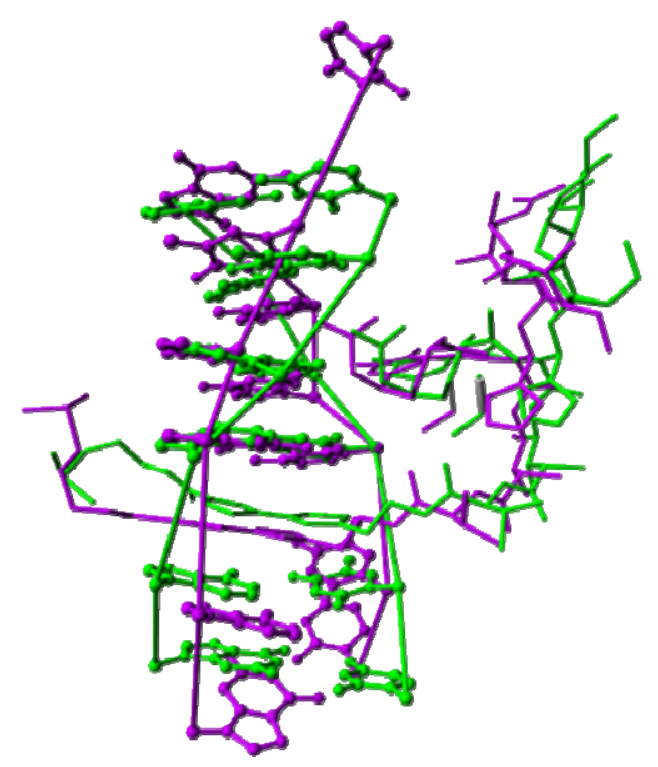

(b)

Figure 3. Superposition of Blm (stick model) and DNA (ball and stick) for the energy minimized initial (green) and final structures of (a) CoBlm (red) and (b) FeBlm (purple). For ease of viewing a simplified representation is used for the DNA sugar-phosphate backbones.
(Figure S4(a)). The terminal tail is oriented more along the DNA helix in the equilibrated structure, but a more extended, horizontal conformation in the final structure (Figure S4(a)). The RMSD values of the heavy atoms between the equilibrated and final structures of DNA-CoBlm for the full system, DNA, and CoBlm are $2.3 \AA, 2.4 \AA$ and $2.1 \AA$, respectively.

Blm does not display any major changes in the DNA-FeBlm complex. RMSDs (heavy atoms) between equilibrated and final structures for the full system, DNA, and Blm are in this case $2.7 \AA, 3.0 \AA$, and $1.9 \AA$, respectively. Although Blm does not undergo any major structural changes, a slight displacement from its initial position is observed. The structural changes in DNA are ascribed to movements in the terminal base pairs (Figure S4(b)).

\subsection{Interactions between BIm and DNA}

The final structures were analysed further with respect to hydrogen bonds between Blm and DNA. In the NMR structure, Blm forms hydrogen bonds through the pyrimidine group of the metal binding domain with N3 and N2 of Guanine G3, and the amide group forms one hydrogen bond with O2 of Cysteine C10 (Figure S2(a)). In the linker domain, the methyl valerate $-\mathrm{OH}$ group forms a hydrogen bond with the O1P phosphate backbone of Thymine T5, and the nitrogen atom in the terminal sulphonium tail forms a hydrogen bond with $\mathrm{O} 6$ of G9 (Figure 4(a), Figures S1 and S3). After MD simulation, the amide group forms a hydrogen bond with $\mathrm{O} 2$ of T11 and also with O3* of the deoxyribose sugar; the remaining H-bonds as outlined above remaining unaltered (Figure 4(b), Figures S1 and S3). FeBlm displays the same $\mathrm{H}$-bonds as described for the CoBlm complex upon MD simulation. In addition, the amide group was also observed to form hydrogen bonds with O1P of the phosphate backbone of T11 and with O2 of C10 (Figure 4(c), Figures S1 and S3).

Both complexes thus retain the hydrogen bonds between the metal binding domain and G3, and between the linker region and T5, as observed in the NMR structure. The hydrogen bond between the terminal sulphonium tail and the phosphate backbone of G9 observed in the NMR structure was not retained in either of the two complexes. This is due to the increased displacement of the tail into the major groove which eventually leads to the loss of the hydrogen bond. No explicit hydrogen bonds were observed between the carbohydrate domain and DNA; however in both complexes close contacts (van der Waals interactions) are seen with the terminal base pairs C12 and G1 (Figure S1).

A closer look at the hydrogen bond distances for the three systems show that these have reduced somewhat 

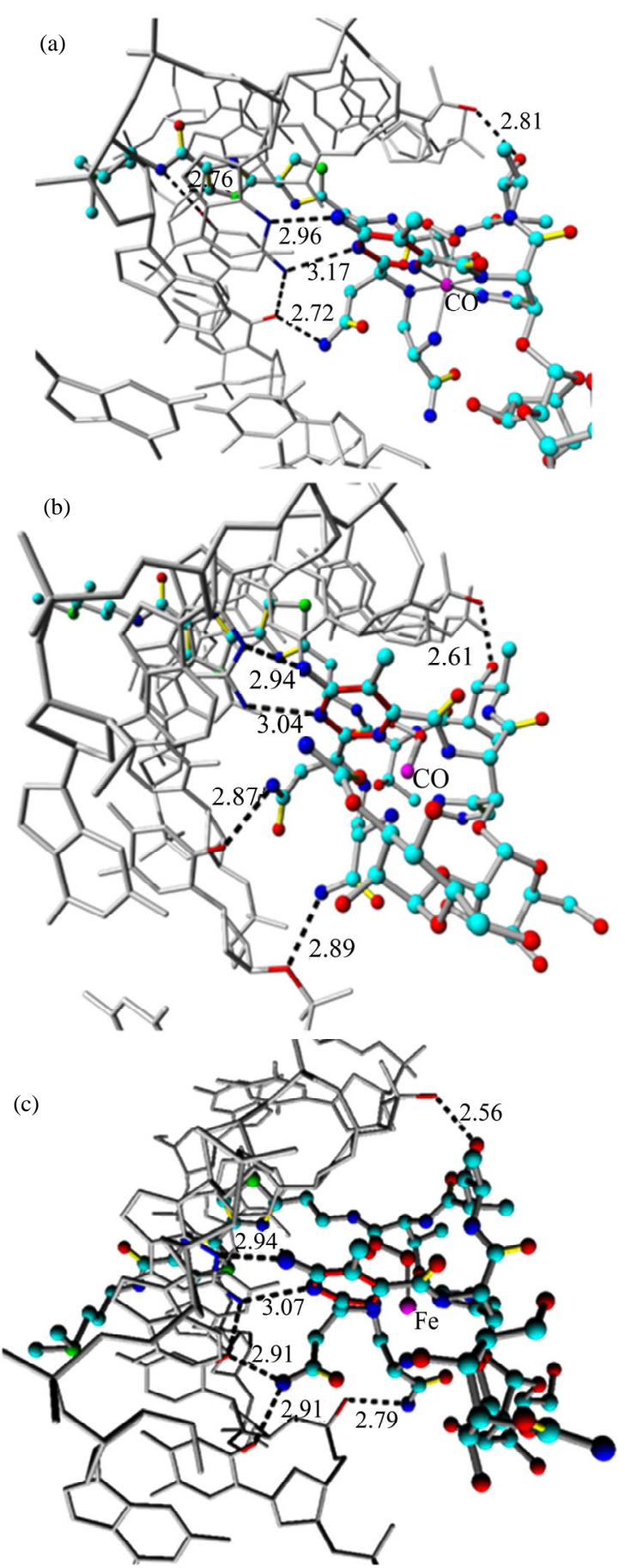

Figure 4. Minor groove view of DNA (grey, stick model) with different Blm complexes (ball and stick) highlighting hydrogen bonds (black dotted lines); (a) NMR structure; (b) CoBlm after MD simulation; (c) FeBlm after MD simulation. Oxygens are show in red and nitrogens in dark blue.

upon MD simulation, compared to the NMR structure; in particular between the linker domain and the backbone of T5. For the NMR structure, CoBlm and FeBlm, this H-bond is $2.81 \AA, 2.61 \AA$ and $2.56 \AA$, respectively Fig- ure 4. This trend also holds for the distances between the pyrimidine group of Blm, and N3 and N2 of G3. These are for the NMR structure $2.96 \AA$ and $3.17 \AA$ (Figure 4(a)), for CoBlm $2.94 \AA$ and $3.04 \AA$ (Figure 4(b)) and for FeBlm $2.94 \AA$ and $3.07 \AA$ (Figure 4(c)), respectively. From the above values it appears that, albeit no major differences are seen, a somewhat stronger interaction is exerted between Blm and DNA after the MD simulations; especially for the FeBlm system.

\subsection{Binding Energies}

Binding energies were computed for the final complexes, using the automated binding energy routine in YASARA; cf. Table 1. This is obtained by calculating the energy of the ligand (MeBlm) and receptor (DNA) at infinite distance, i.e., when there is zero interaction between them and subtracting the obtained value from the energy of their bound state (complex). The final complexes were energy minimized before calculating binding energies. Water was removed and the complexes were placed in a simulation cell with rigid walls, as it is not possible to move ligand and receptor to infinite distances in a periodic boundary cell.

FeBlm shows a significantly higher binding energy than CoBlm. This can be attributed to the increased number of hydrogen bonds exerted by FeBlm, and that it is tighter bound to DNA as manifested through the shorter hydrogen bonds. The stronger interaction energy between FeBlm and DNA, compared to CoBlm, also provides a setting for its enhanced reactivity.

\subsection{Hydrogen Abstraction}

According to the direct mechanism (cf. Introduction), Blm induced DNA strand cleavage starts with the abstraction of a hydrogen atom from C4' of deoxyribose by the metal-bound peroxide group of the activated $\mathrm{Blm}$. The distances between the distal peroxide oxygen and C4' and H4' of cytosine $\mathrm{C} 4$, which is the pyrimidine most accessible for Blm to abstract a hydrogen from, were measured (Figure 5). The distances between C4', H4' and the distal oxygen atom are $4.81 \AA$ and $4.21 \AA$, respectively, in the initial structure (Figure 5). In both complexes, the distances were reduced after the MD simulations, to the values $4.60 \AA$ and $4.13 \AA$ (CoBlm), and $4.01 \AA$ and $3.28 \AA$ (FeBlm), respectively.

During the MD simulations the Blm molecule thus positions itself in closer proximity to C4', making it much more favorably disposed to abstract the hydrogen. From the above values FeBlm (Figure 5(c)) is closer to C4' by $0.5 \AA$, compared to CoBlm (Figure 5(b)), which in turn also explains the higher reactivity of the former. The short distance ( $3.3 \AA$ ) furthermore supports the direct mecha- 

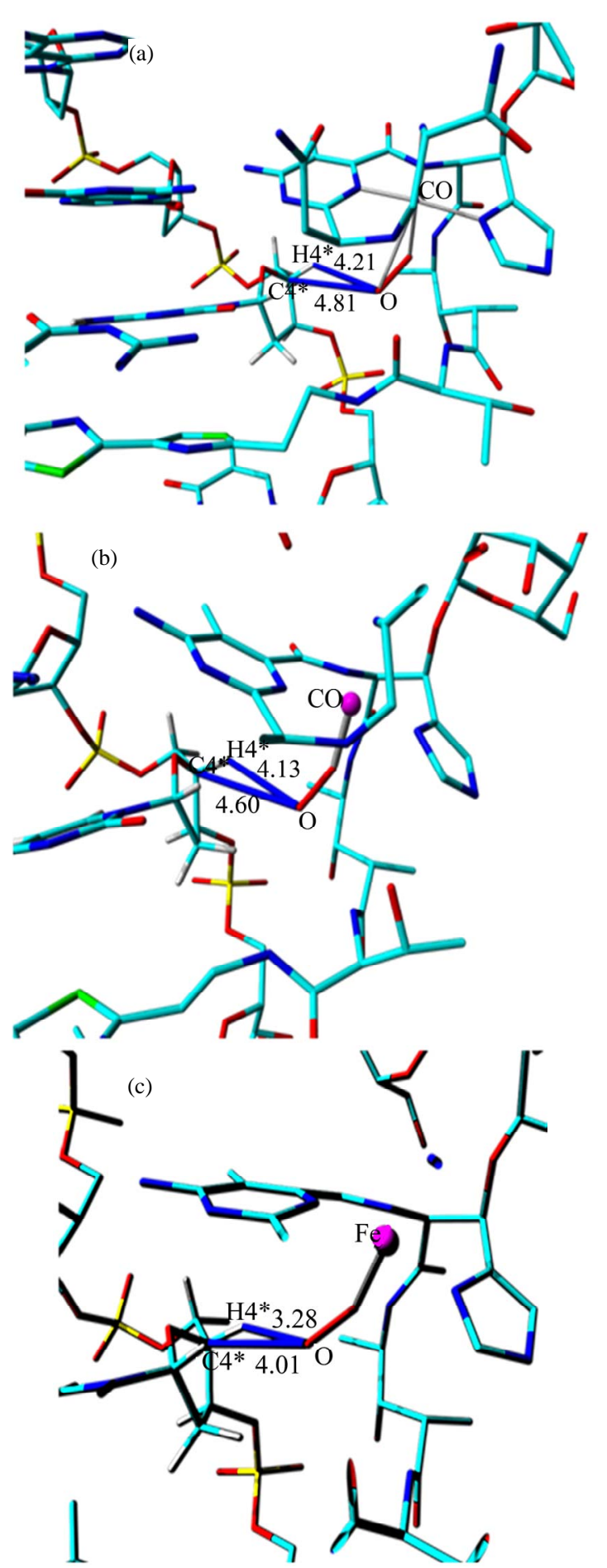

Figure 5. Distance between C4' and H4' of C4 in DNA, and the distal peroxide oxygen atom of Blm. (a) NMR Structure; (b) CoBlm final structure; (c) FeBlm final structure.

nism proposed by Solomon and coworkers [22,24].

The distances between the distal peroxide oxygen atom and C4' and $\mathrm{H} 4$ ' were furthermore monitored during the MD simulations. In the CoBlm complex, the distances to the distal peroxide oxygen increase slightly in the equilibration phase of the MD simulation, whereafter a minor reduction is observed after which the distance stays highly stable for the remainder of the simulation (Figure 6(a)). On average the distances in CoBlm stay close to the crystal structure values (Figures 5(a) and (b)), with an average $\mathrm{O}-\mathrm{H} 4$ ' distance in the production run of $3.25 \pm 0.5 \AA$. The FeBlm complex, on the other hand, shows slightly larger fluctuations in the $\mathrm{O}-\mathrm{H} 4$ ' and $\mathrm{O}-\mathrm{C} 4$ ' distances. In the beginning of production phase of the MD simulation a maximum of 6 $\AA$ (5 $\AA$ ) between C4' (H4') and the distal oxygen atom is noted. An increase in the distances, albeit smaller, is also observed in the end of the production run. The maximum distances shown by the FeBlm complex differ from the CoBlm NMR structure data by $1 \AA$ (0.8 $\AA$ ) for C4' (H4'). However, the initial large fluctuation is observed only for a very short period at around 6 ns. The average $\mathrm{O}-\mathrm{H} 4$ ' value during the production run (excluding the final 2ns) is $2.90 \pm 0.5 \AA$; i.e. considerably shorter than for CoBlm.

\subsection{Electrostatic Potential Maps}

Electrostatic potential maps of DNA were generated using Poisson-Boltzmann Solver (ESPPBS) for the initial NMR structure and the final structures after MD. We first of all note that the charge distribution around the intercalated bithiazole region in the NMR structure (Figure S5(a)) is denser compared to the other two complexes. At the end of the MD simulations, the distance between the DNA strands have increased, resulting in reduced charge distribution around the intercalated region. We furthermore note that the charge distribution in the minor groove around the metal binding domain is more negative (smaller blue patches) in the case of the FeBlm complex (Figure S5(c)) compared to CoBlm (Figure S5(b)), to the positive metal ion. This may also be one contributing factor for the noted higher binding energy of the FeBlm complex.

\section{CONCLUSIONS}

The NMR structure of CoBlm complexed to a DNA hexamer duplex is used as a model system to study the properties and structural characteristics of biologically active FeBlm and how these differ from the less potent CoBlm system. Based on 25 ns MD simulations of DNA-FeBlm and DNA-CoBlm, the following conclusions can be made. The RMSD values show that DNA undergoes larger structural changes when complexed with FeBlm compared to CoBlm. FeBlm furthermore interacts much stronger with DNA, as seen in the increased number of hydrogen bonds to DNA, the shorter hydrogen bond distances, the higher binding energy, and 


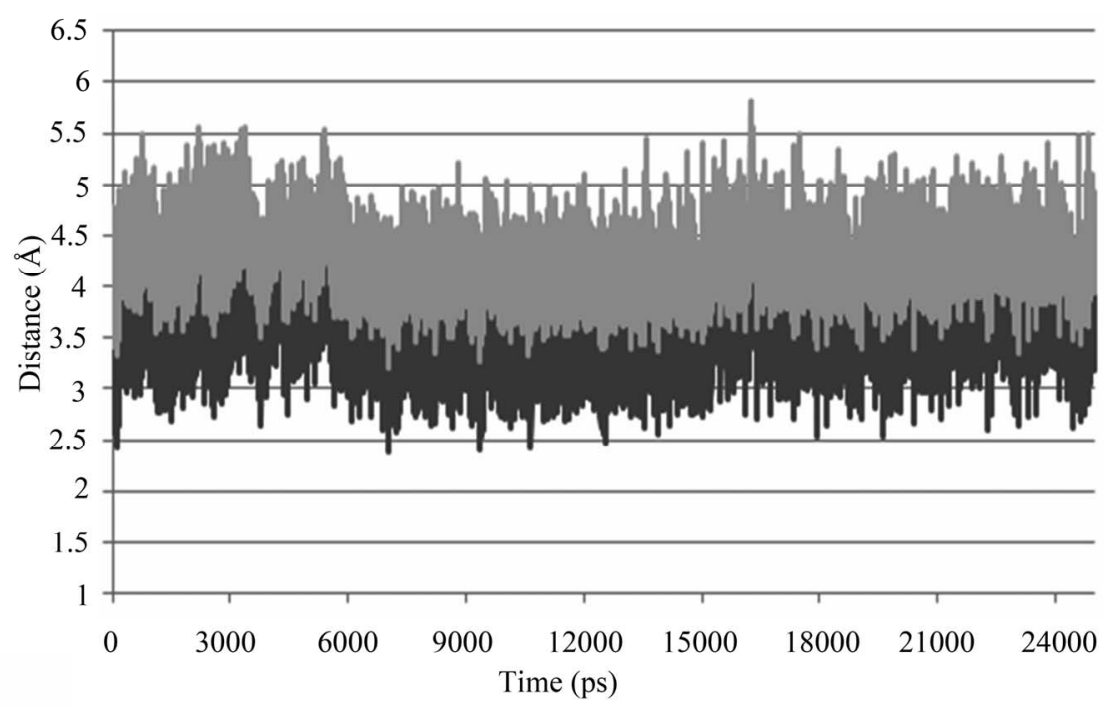

(a)

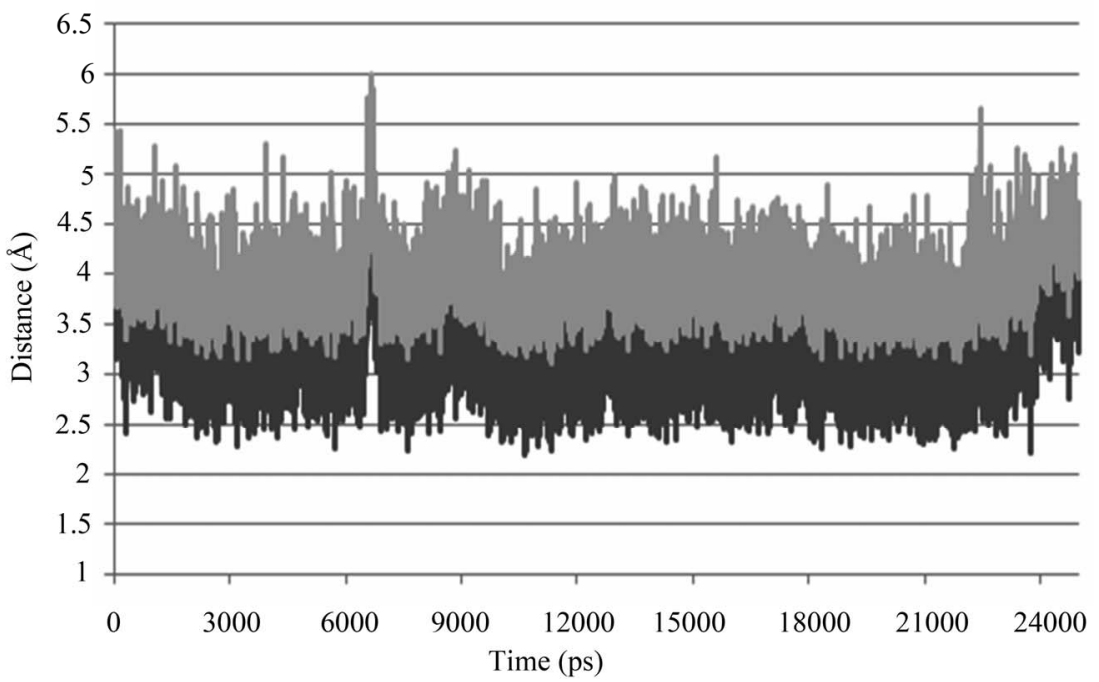

(b)

Figure 6. Distance between distal peroxide oxygen atom and H4' (black) and C4' (grey) of C4 in DNA versus time during the MD simulation in (a) CoBlm complex and (b) FeBlm complex.

the favourable electrostatics. The metal-bound peroxide moiety of activated Blm, responsible for the initiation of DNA strand cleavage through abstraction of a pyrimidine nucleotide deoxyribose hydrogen (H4') is located more than $0.5 \AA$ closer to H4' in FeBlm than what is the case for CoBlm. All the above data provides evidence for the stronger affinity of DNA towards FeBlm, and the higher reactivity thereof. The close proximity between $\mathrm{OOH}$ and H4' supports the direct mechanism as proposed by Solomon and coworkers [22,24].

\section{ACKNOWLEDGEMENTS}

The faculty of science and technology at Örebro University, the Swedish Science Research Council (VR), and the National university of Ireland-Galway are gratefully acknowledged for financial support.

\section{SUPPORTING INFORMATION}

Superposed initial vs final and equilibrated vs final structures of the DNA-CoBlm and DNA-FeBlm systems, electrostatic maps, and structure and labeling of atoms and nucleotides used in the present study are available via http://www.scirp.journal/JBPC

\section{REFERENCES}

[1] Cragg, G.M., Newman, D.J. and Snader, K.M. (1997) Natural products in drug discovery and development. Journal of Natural Products, 60, 52-60. doi:10.1021/np9604893

[2] Kopka, M.L., Yoon, C., Goodsell, D., Pjura, P. and Dickerson, R.E. (1985) The molecular origin of DNA-drug specificity in netropsin and distamycin. Proceedings National Academy Sciences USA, 82, 
1376-1380. doi:10.1073/pnas.82.5.1376

[3] Tse, W.C. and Boger, D.L. (2004) Sequence-selective DNA recognition: Natural products and nature's lessons. Chemical Biology, 11, 1607-1617.

doi:10.1016/j.chembiol.2003.08.012

[4] Wang, A.H., Ughetto, G., Quigley, G.J. and Rich, A. (1987) Interactions between an anthracycline antibiotic and DNA: Molecular structure of daunomycin complexed to d(CpGpTpApCpG) at 1.2- $\AA$ resolution. Biochemistry, 26, 1152-1163. doi:10.1021/bi00378a025

[5] Umezawa, H., Maeda, K., Takeuchi, T. and Okami, Y. (1966) New antibiotics, bleomycin A and B. The Journal of Antibiotic, 19, 200-209.

[6] Blum, R.H., Carter, S.K. and Agre, K. (1973) A clinical review of bleomycin-A new antineoplastic agent. Cancer, 31, 903-914.

doi:10.1002/1097-0142(197304)31:4<903::AID-CNCR2 $820310422>3.0 . \mathrm{CO} ; 2-\mathrm{N}$

[7] Umezawa, H. (1974) Chemistry and mechanism of action of bleomycin. Federal Process, 33, 2296-2302.

[8] Stubbe, J.A., Kozarich, J.W., Wu, W. and Vanderwall, D.E. (1996) Bleomycins: A structural model for specificity, binding, and double strand cleavage. Accounts of Chemical Research, 29, 322-330. doi:10.1021/ar9501333

[9] Boger, D.L., Ramsey, T.M., Cai, H., Hoehn, S.T. and Stubbe, J.A. (1998) A systematic evaluation of the bleomycin A(2) L-threonine side chain: Its role in preorganization of a compact conformation implicated in sequence-selective DNA cleavage. Journal of the American Chemical Society, 120, 9139-9148. doi:10.1021/ja9816638

[10] Tanaka, W. (1977) Development of new bleomycins with potential clinical utility. Japanese Journal of Antibiotics, 30, 41-48.

[11] Boger, D.L. and Cai, H. (1999) Bleomycin: Synthetic and mechanistic studies. Angewandte Chemie International Edition, 38, 448-476.

doi:10.1002/(SICI)1521-3773(19990215)38:4<448::AID -ANIE448>3.0.CO;2-W

[12] Hecht, S.M. (2000) Bleomycin: New perspectives on the mechanism of action. Journal of Natural Products, 63, 158-168. doi:10.1021/np990549f

[13] Stubbe, J.A. and Kozarich, J.W. (1987) Mechanisms of bleomycin-induced DNA-degradation. Chemical Reviews, 87, 1107-1136. doi:10.1021/cr00081a011

[14] Burger, R.M., Peisach, J. and Horwitz, S.B. (1982) Effects of $\mathrm{O}_{2}$ on the reactions of activated bleomycin. Journal of Biological Chemistry, 257, 3372-3375.

[15] Breen, A.P. and Murphy, J.A. (1995) Reactions of oxyl radicals with DNA. Free Radical Biology and Medicine, 18, 1033-1077. doi:10.1016/0891-5849(94)00209-3

[16] Wu, W. et al. (2002) Solution structure of the hydroperoxide of $\mathrm{Co}(\mathrm{III})$ phleomycin complexed with d(CCAGGCCTGG)2: Evidence for binding by partial intercalation. Nucleic Acids Research, 30, 4881-4891. doi:10.1093/nar/gkf608

[17] Zhao, C. et al. (2002) Structures of HO(2)-Co(III)bleomycin $\mathrm{A}(2)$ bound to d(GAGCTC)(2) and d(GGAAGC TTCC)(2): Structure-reactivity relationships of Co and Fe bleomycins. Journal of Inorganic Biochemistry, 91 259-268. doi:10.1016/S0162-0134(02)00420-8
[18] Papakyriakou, A., Mouzopoulou, B. and Katsaros, N. (2003) The solution structure of the Ga(III)-bleomycin A2 complex resolved by NMR and molecular modeling; interaction with d(CCAGGCCTGG). Journal of Biological Inorganic Chemistry, 8, 549-459.

[19] Lehmann, T.E., Ming, L.J., Rosen, M.E. and Que Jr, L. (1997) NMR studies of the paramagnetic complex Fe(II)-bleomycin. Biochemistry, 36, 2807-2816. doi:10.1021/bi962748t

[20] Lin, P.S., Kwock, L., Hefter, K. and Misslbeck, G. (1983) Effects of iron, copper, cobalt, and their chelators on the cytotoxicity of bleomycin. Cancer Research, 43, 1049-1053.

[21] Rao, E.A., Saryan, L.A., Antholine, W.E. and Petering, D.H. (1980) Cytotoxic and antitumor properties of bleomycin and several of its metal complexes. Journal of Medicinal Chemistry, 23, 1310-1318. doi:10.1021/jm00186a006

[22] Decker, A., Chow, M.S., Kemsley, J.N., Lehnert, N. and Solomon, E.I. (2006) Direct hydrogen-atom abstraction by activated bleomycin: An experimental and computational study. Journal of the American Chemical Society, 128, 4719-4733. doi:10.1021/ja057378n

[23] Kumar, D., Hirao, H., Shaik, S. and Kozlowski, P.M. (2006) Proton-shuffle mechanism of O-O activation for formation of a high-valent oxo-iron species of bleomycin. Journal of the American Chemical Society, 128, 16148-16158. doi:10.1021/ja0646110

[24] Chow, M.S., Liu, L.V. and Solomon, E.I. (2008) Further insights into the mechanism of the reaction of activated bleomycin with DNA. Proceedings of National Academy Sciences USA, 105, 13241-132415. doi:10.1073/pnas.0806378105

[25] Karawajczyk, A. and Buda, F. (2005) The metal bonding domain of the antitumor drug Fe(II)-bleomycin: A DFT investigation. Journal of Biological Inorganic Chemistry, 10, 33-40. doi:10.1007/s00775-004-0610-8

[26] Krieger, E., Darden, T., Nabuurs, S.B., Finkelstein, A. and Vriend, G. (2004) Making optimal use of empirical energy functions: Force-field parameterization in crystal space. Proteins: Structure, Function, Bioinformatics, 57, 678-683.

[27] Zhao, C. et al. (2002) Structures of HO2-Co(III) bleomycin $\mathrm{A}_{2}$ bound to d(GAGCTC)2 and d(GGAA GCTTCC)2: Structure-reactivity relationships of Co and Fe bleomycins. Journal of Inorganic Biochemistry, 91, 259-268. doi:10.1016/S0162-0134(02)00420-8

[28] Duan, Y. et al. (2003) A point-charge force field for molecular mechanics simulations of proteins based on condensed-phase quantum mechanical calculations. Journal of Computational Chemistry, 24, 1999-2012. doi:10.1002/jcc.10349

[29] Essmann, U., Perera, L., Berkowitz, M.L., Darden, T., Lee, H. and Pedersen, L.G. (1995) A smooth particle mesh ewald method. Journal of Chemical Physics, 103, 8577-8593. doi:10.1063/1.470117

[30] Jorgensen, W.L. (1981) Quantum and statistical mechanical studies of liquids .10. Transferable intermolecular potential functions for water, alcohols, and ethers-Application to liquid water. Journal of the American Chemical Society, 103, 335-340. doi:10.1021/ja00392a016 


\section{SUPPLEMENTARY MATERIAL}

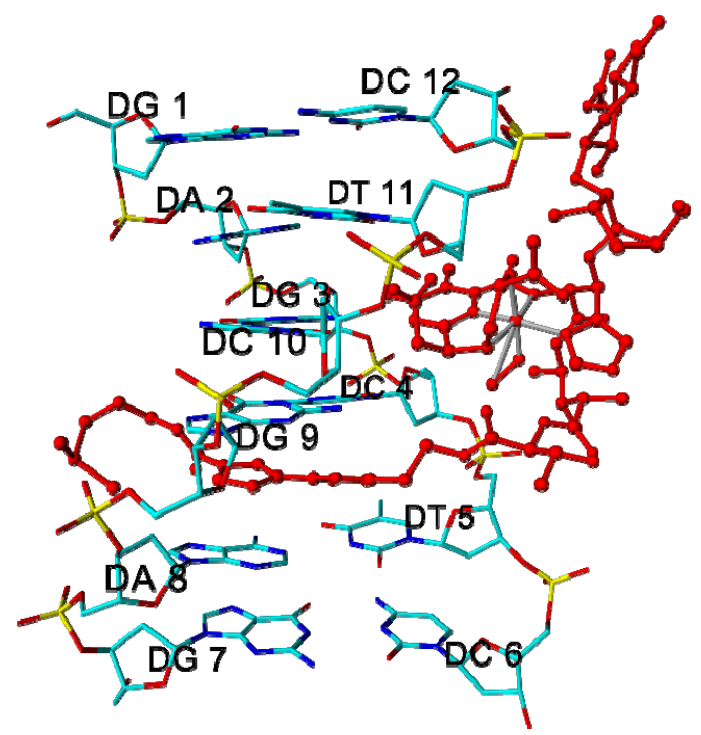

Figure S1. NMR structure showing DNA (stick) and Blm in red (ball and stick). 


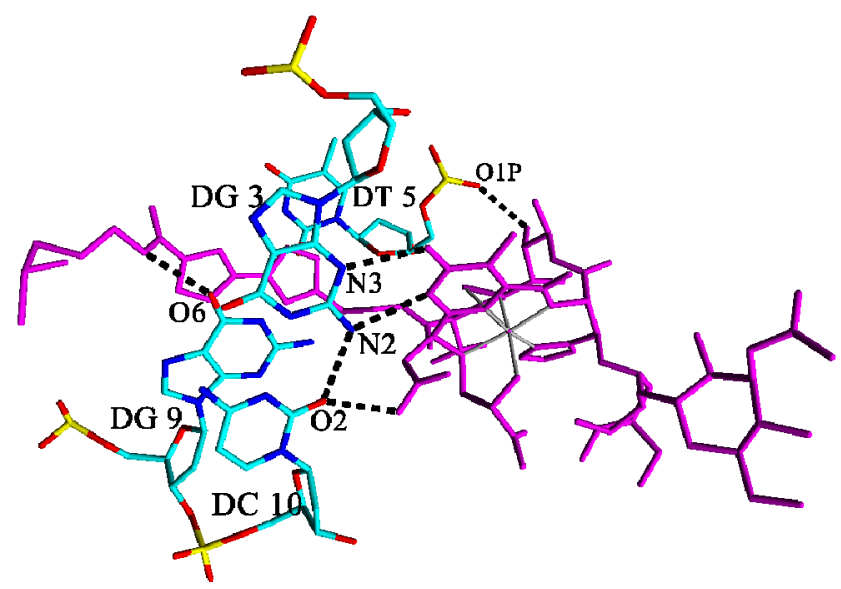

(a)

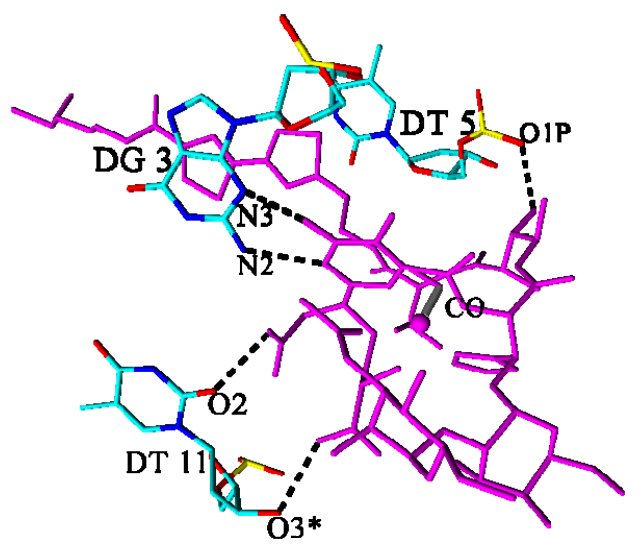

(b)

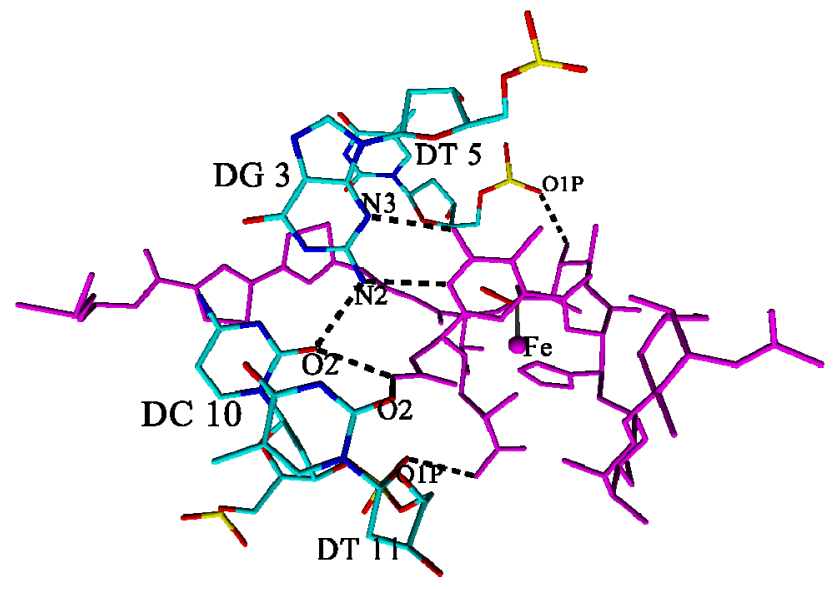

(c)

Figure S2. Hydrogen bonds (black dotted lines) between DNA and MeBlm (stick, magenta) of (a) NMR structure (b) CoBlm after MD simulation (c) FeBlm after MD simulation. Only DNA residues involved in hydrogen bonds are shown. Only DNA residue atoms involved in hydrogen bonds are labeled. Hydrogens are not shown, to help in easy representation. 


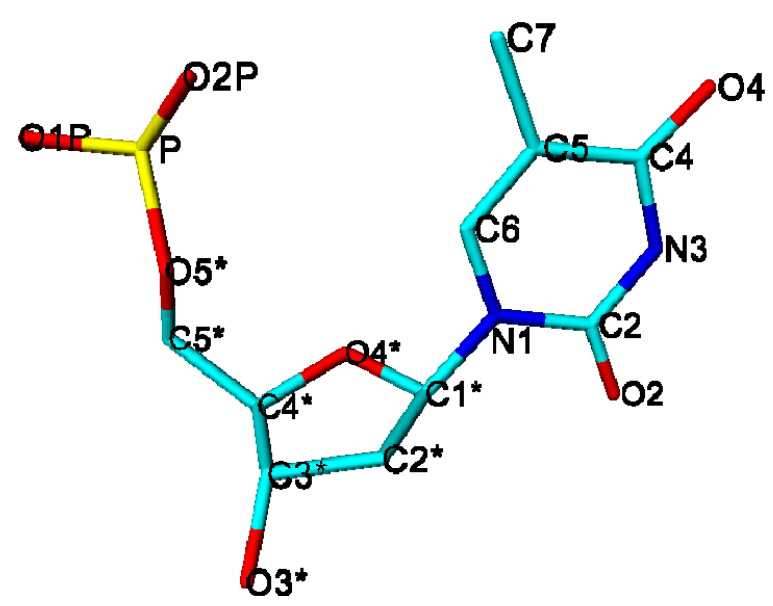

DT11

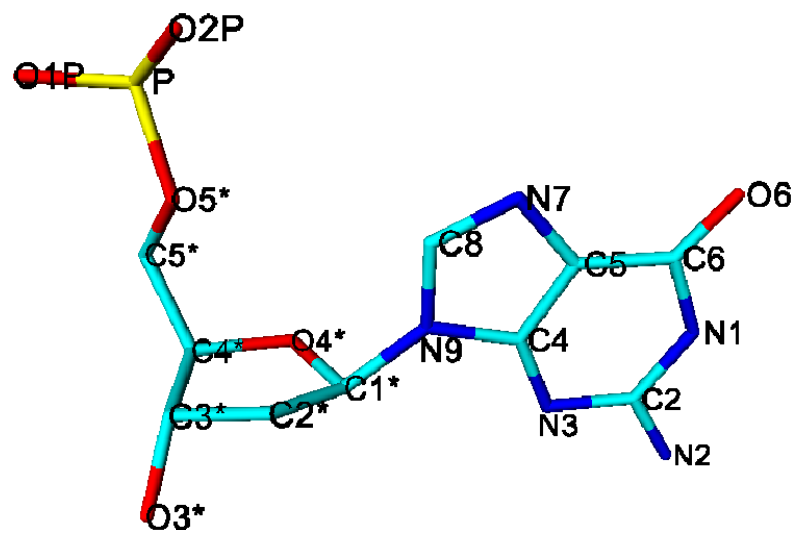

DG3

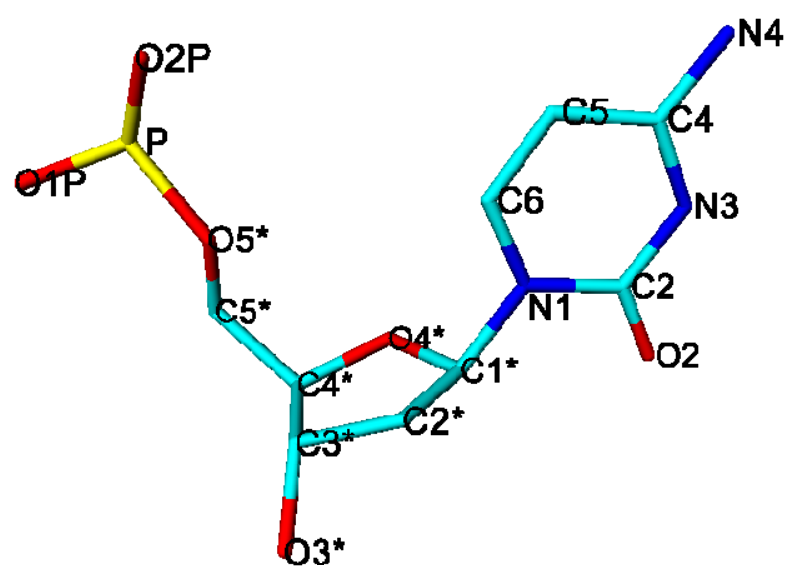

DT10

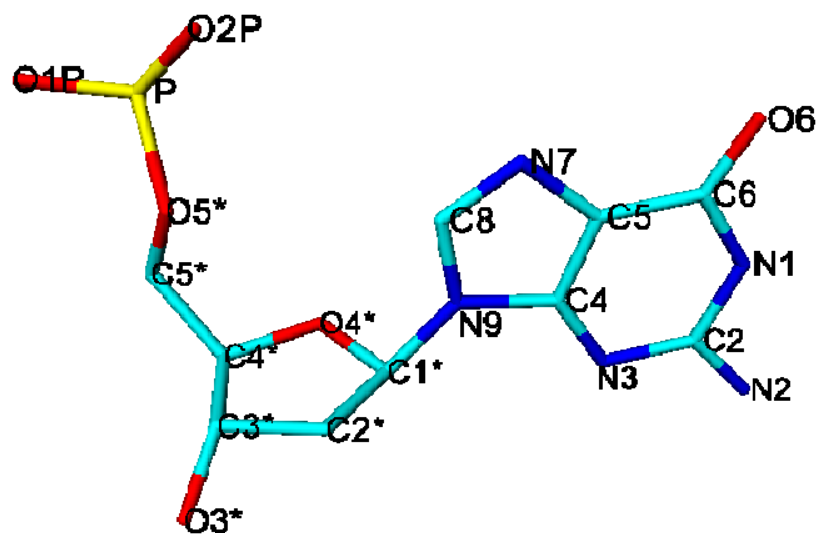

DG9

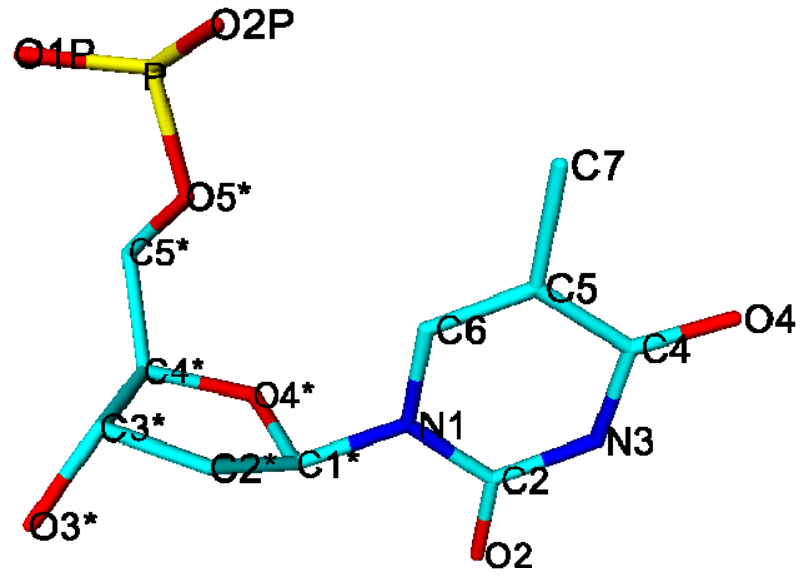

DT5

Figure S3. Structure of nucleotides (heavy atoms) along with the atom names used in the present study. Only DNA residues involved in hydrogen bonding with MeBlm are shown.SS. 


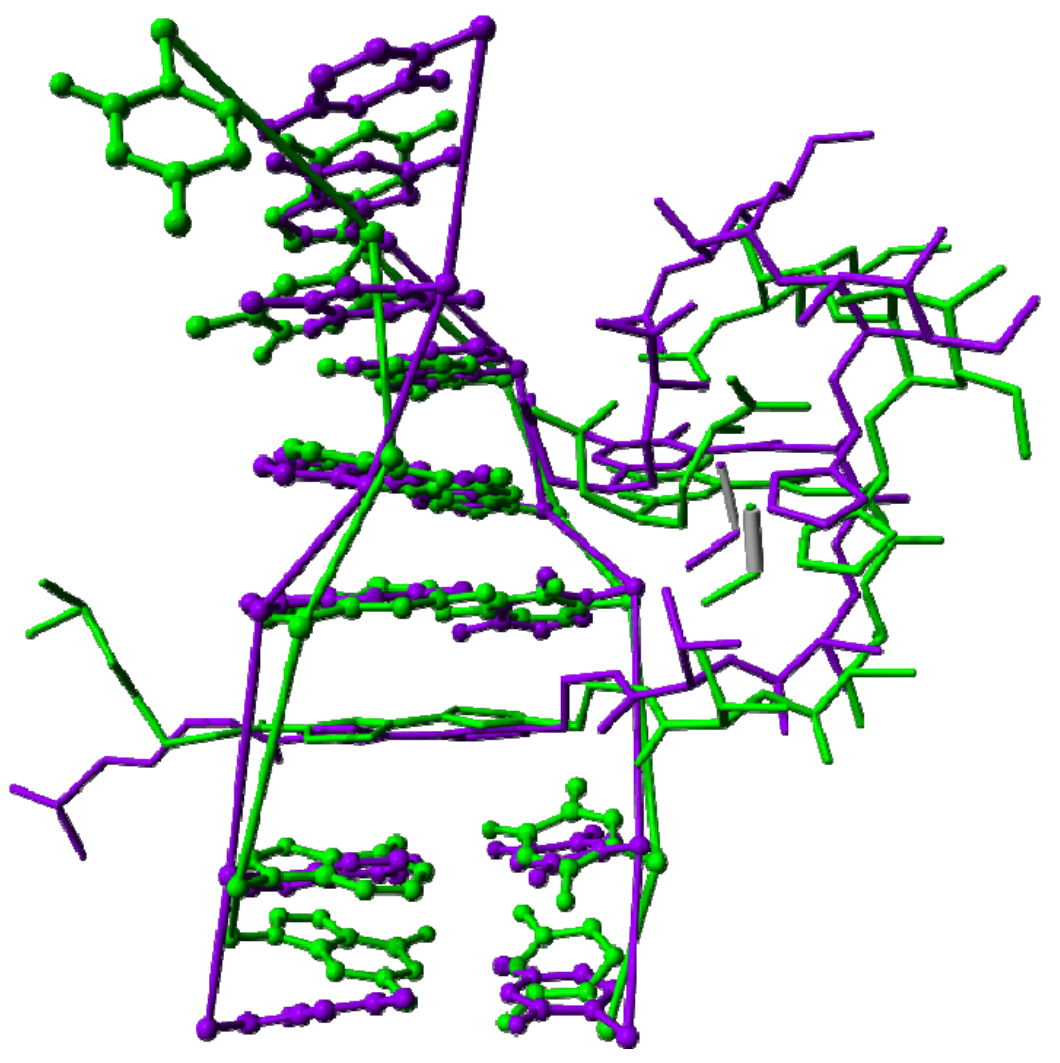

(a)

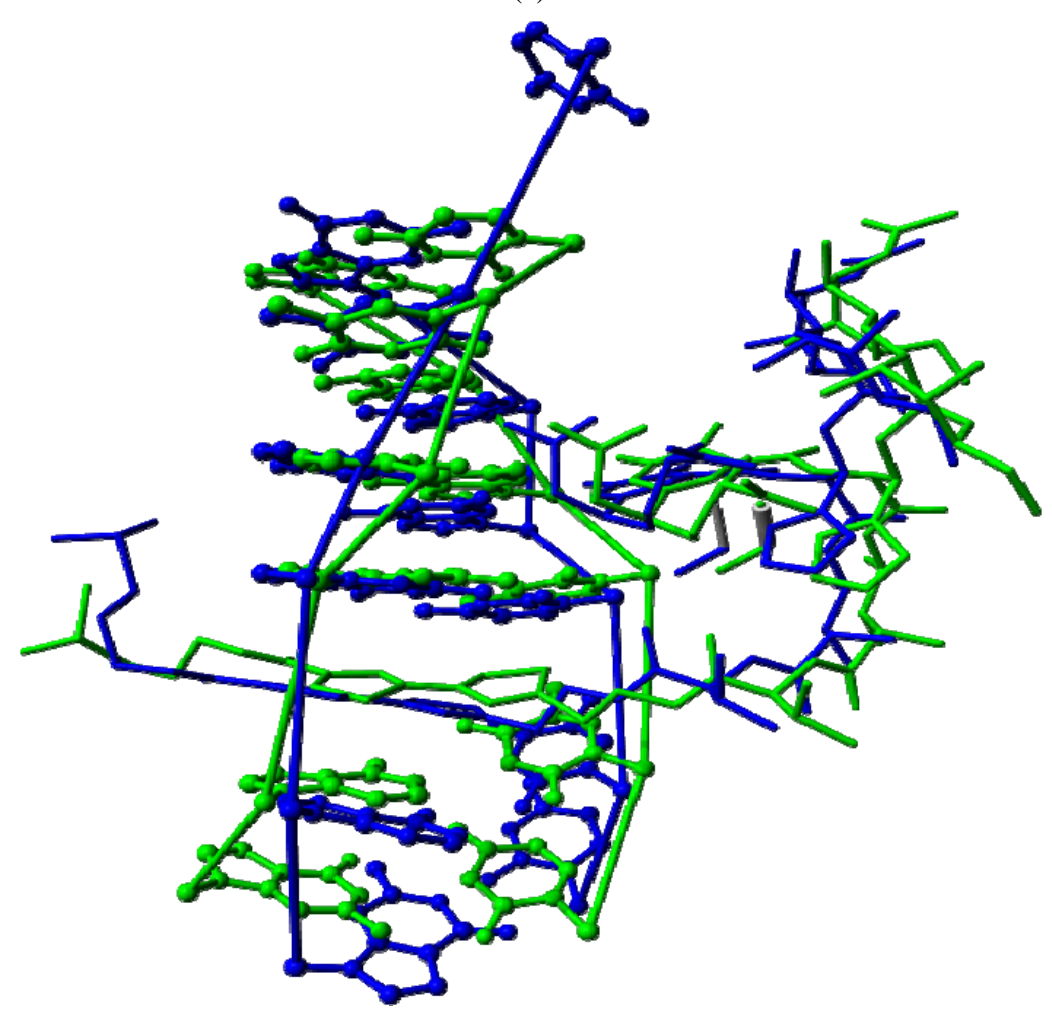

(b)

Figure S4. Superposed structures of equilibrated (green) and final structures of (a) CoBlm (purple) (b) FeBlm (blue). 


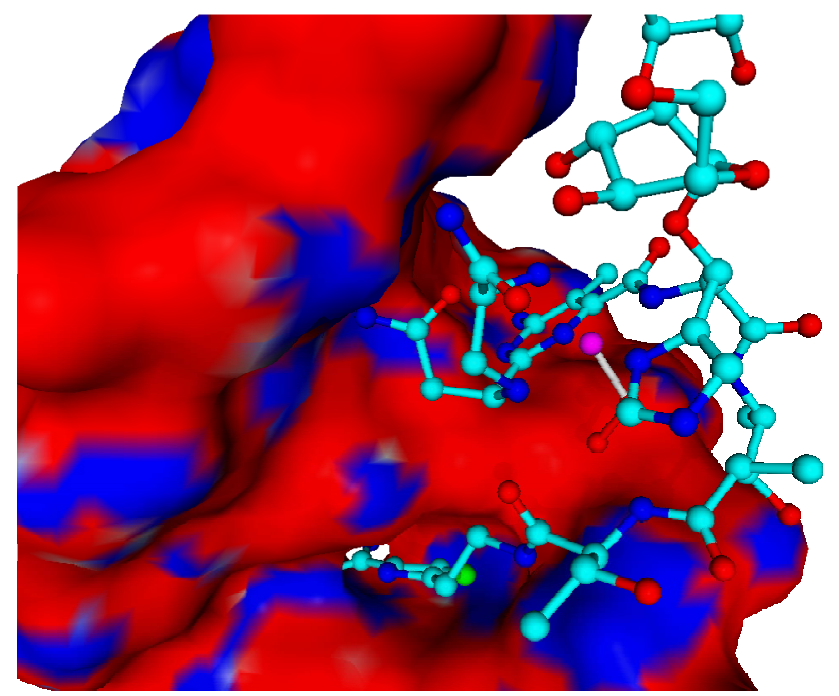

(a)

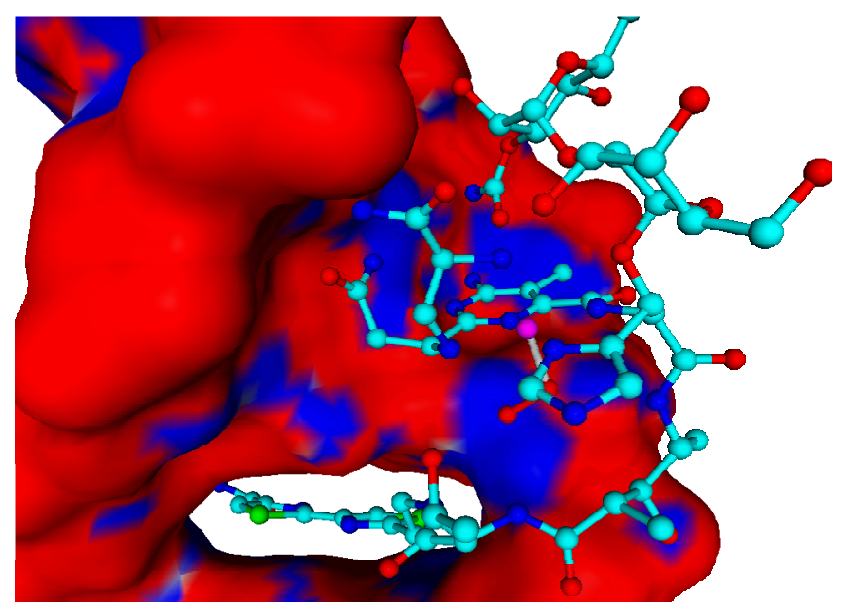

(b)

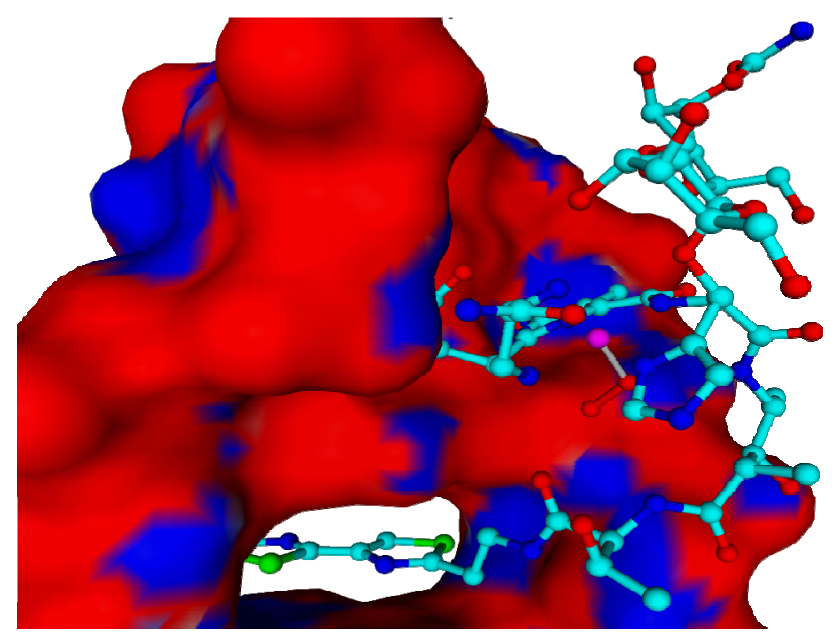

(c)

Figure S5. Electrostatic potential map of (a) NMR structure; (b) CoBlm final structure; (c) FeBlm final structure. Charge distribution is represented in blue for positive charge and red for negative charge. 